\title{
Bortezomib Comes to the Rescue: Comment on the Article by Nachmias et al. Entitled "A Bortezomib- Based Protocol Induces a High Rate of CR with Minor Toxicity in Adult Patients with Relapsed/ Refractory ALL"
}

\author{
Yoav H. Messinger Bruce C. Bostrom \\ Cancer and Blood Disorders, Children's Hospitals and Clinics of Minnesota, Minneapolis, MN, USA
}

In this issue of Acta Haematologica, Nachmias et al. [1] confirm that in adult patients with relapsed/refractory acute lymphoblastic leukaemia (ALL) adding bortezomib to a classical 4-drug induction regimen allows for a high rate of remission.

Bortezomib, a first-generation proteasome inhibitor has shown remarkable activity for mature plasma-cell/ B-cell malignancies and is FDA-approved for multiple myeloma and mantle-cell lymphoma. There was ample preclinical data for single-agent activity in ALL, but single-agent clinical trials showed no activity in patients with ALL, which is not surprising as bortezomib is only active as a single agent in myeloma. Horton et al. [2] were the first to describe that bortezomib has a synergistic or additive effect with traditional chemotherapy agents.

Brown et al. [3] recognized the unique activity of bortezomib combined with dexamethasone and showed remarkable but transient activity in a relapsed ALL patient. This was followed by our own observation that bortezomib and a standard 4-drug combination had remarkable activity in relapsed/refractory childhood B-cell ALL [4]. This has since been confirmed by other studies on childhood ALL, with activity even being seen in childhood Tcell ALL (Table 1).

\section{KARGER}

(c) 2018 S. Karger AG, Basel

E-Mail karger@karger.com

www.karger.com/aha
Although the outcome of ALL in adults has improved with the use of paediatric-type regimens, the number of relapsing patients remains high and their outcome remains profoundly poor. Moreover, as noted by Gaynon and Sun [5], relapsed ALL, as opposed to primary ALL, has different clones that require different agents or approaches. This led Nachmias et al. [1] from Israel to try the bortezomib-VXLD (B-VXLD) regimen in 9 adults with relapsed or refractory ALL.

They confirm the results shown for children with an overall remission induction (CR/CRi) of 7/9 (78\%) patients. The response was better in B-cell ALL 5/5 (100\%) than in T-cell ALL 2/4 (50\%). Combining the 3 studies, including that of Nachmias et al. [1], the response to BVXLD regimen in B-cell ALL (78\%) is not significantly different from that in T-cell ALL (69\%) (Table 1, $p=0.49$ ). Combining response data from 5 studies using bortezomib and standard chemotherapy for advanced relapsed/ refractory ALL in 169 children and adults, the CR/Cri rate is $71 \%$ (Table 1 ) which is better than the $44 \%$ benchmark developed for paediatrics for $\geq 2$ relapses [6].

Nachmias et al. [1] report a rate of toxicity in adults that is not at all different from that in children. Although both vincristine and bortezomib are known to cause pe-

Yoav H. Messinger

Cancer and Blood Disorders, Children's Hospitals and Clinics of Minnesota 2530 Chicago Ave. S.

Minneapolis, MN 55404 (USA)

E-Mail yoav.messinger@childrensmn.org 
Table 1. Studies using bortezomib combined with chemotherapy for relapsed/refractory ALL in adults and children

\begin{tabular}{lccc}
\hline Regimen (response) & All patients & B-cell ALL patients & T-cell ALL patients \\
\hline B-VXLD & & & \\
$\quad$ Messinger et al. [4] (CR + CRp) & $16 / 22(73)$ & $16 / 20(80)$ & $0 / 2(0)$ \\
$\quad$ Bertaina et al. [7] (CR + CRp) & $27 / 37(73)$ & $22 / 30(73)$ & $5 / 7(71)$ \\
$\quad$ Nachmias et al. [1] (CR + CRi) & $7 / 9(77)$ & $5 / 5(100)$ & $2 / 4(50)$ \\
Total & $\mathbf{5 0 / 6 8 ( 7 4 )}$ & $\mathbf{4 3 / 5 5 ( 7 8 )}$ & $\mathbf{9 / 1 3 ( 6 9 )}$ \\
\hline B-VPLD & $62 / 92(67)$ & 70 (n.a.) & (n.a.) \\
$\quad$ Horton et al. [10] & & 6 (n.a.) & 3 (n.a.) \\
\hline B-hyper-CVAD or B-HD-MTX/Ara-C & & & \\
$\quad$ Zhao et al. [11] (CR) & $\mathbf{1 2 0 / 9 ( 8 9 )}$ & $(\mathbf{7 1 )}$ & \\
\hline Total (CR + CRi) & & & \\
\hline
\end{tabular}

Values are expressed as $n / N(\%)$. ALL, acute lymphoblastic leukaemia; B-VXLD, bortezomib + vincristine + dexamethasone + PEG-asparaginase + doxorubicin; B-VPLD, bortezomib + vincristine + prednisone + PEGasparaginase + doxorubicin; B-hyper-CVAD, bortezomib + fractionated cyclophosphamide + vincristine + doxorubicin + dexamethasone; B-HD-MTX/Ara-C, bortezomib + high-dose methotrexate + cytarabine; CR, complete remission; $\mathrm{CRp}$, complete remission without platelet recovery; CRi, CR incomplete (i.e., CR except for ANC and/or platelet recovery); n.a., response not available.

${ }^{\mathrm{a}} \mathrm{B}$-VXLD (CR + CRi): B-cell ALL versus T-cell ALL; $\chi^{2}=0.47, p=0.49$, ns.

ripheral neuropathy, this was not a significant problem in any studies to date. Interestingly, though, among these heavily treated adults, only 2 bacterial infections were reported and no significant sepsis, in contrast to the high concern for bacterial and fungal infections raised by the TACL group (3 infectious deaths) [4] and the European group (3 infectious deaths) [7].

Nachmias et al. [1] should be congratulated on their careful management of the intensive PEG-asparaginase used in this regimen (a significant concern in adults); they gave it to $7 / 9$ patients $<50$ years of age with no undue toxicity. Whether it can be omitted as was done in 2 of the patients who attained remission remains unclear. Notably, carnitine can be used to prevent haptic toxicity in patients with fatty liver disease and it may reduce PEGasparaginase hepatotoxicity [8]. Additionally, Erwinia asparaginase can be used if a patient has a history of allergy to PEG-asparaginase.

The landscape of approved therapies used for relapsed/ refractory B-cell ALL has changed dramatically with the use of CD19- or CD22-CAR T cells. The CD22-directed inotuzumab and the CD19-directed blinatumomab are also approved for relapsed/refractory B-cell ALL. Thus, it is unlikely that new agents or chemotherapy-based combinations for B-cell ALL have a place, unless patients become CD19- and CD22-negative. This problem is emerg- ing as more patients are exposed to this targeted therapy. In T-cell disease, there are no new agents on the horizon, and relapsed T-cell ALL is almost uniformly fatal with a profoundly low rate of response. With the caveat that a low number of T-cell ALL patients have been treated with B-VXLD, bortezomib-containing regimens do seem to be active in a high enough number to warrant using this regimen for relapsed/refractory T-cell ALL.

Finally, predicting resistance to bortezomib using a biomarker may help in tailoring this therapy more precisely. This is because we assume that, in most cases, the relapsed refractory clone is resistant, at least to the steroid component. It is possible that in ALL, the ratio of the immunoproteasome to constitutive proteasome subunit expression might predict an early response to bortezomibcontaining regimens, as evaluated by the group from The Netherlands [9]. It is worth noting, however, that they found no evidence that NF- $\kappa B$ correlates with the response, so NF- $\mathrm{kB}$ may not be a biomarker that is worth pursuing.

\section{Disclosure Statement}

The authors have no conflicts of interest to declare. 


\section{References}

1 Nachmias B, Shaulov A, Gatt ME, Shapira M, Gural A. A Bortezomib-based protocol induces a high rate of $\mathrm{CR}$ with minor toxicity in adult patients with relapsed/refractory ALL. Acta Haematol. 2018;140:209-14.

2 Horton TM, Gannavarapu A, Blaney SM, D’Argenio DZ, Plon SE, Berg SL. Bortezomib interactions with chemotherapy agents in acute leukemia in vitro. Cancer Chemother Pharmacol. 2006 Jul;58(1):13-23.

3 Brown RE, Bostrom B, Zhang PL. Morphoproteomics and bortezomib/dexamethasoneinduced response in relapsed acute lymphoblastic leukemia. Ann Clin Lab Sci. 2004; 34(2):203-5.

4 Messinger YH, Gaynon PS, Sposto R, van der Giessen J, Eckroth E, Malvar J, et al; Therapeutic Advances in Childhood Leukemia \& Lymphoma (TACL) Consortium. Bortezomib with chemotherapy is highly active in advanced B-precursor acute lymphoblastic leu- kemia: Therapeutic Advances in Childhood Leukemia \& Lymphoma (TACL) Study. Blood. 2012 Jul;120(2):285-90.

5 Gaynon PS, Sun W. Oligoclonality and new agent evaluation in acute lymphoblastic leukaemia. Br J Haematol. 2016 Jun;173(6): 950-7.

6 Ko RH, Ji L, Barnette P, Bostrom B, Hutchinson R, Raetz E, et al. Outcome of patients treated for relapsed or refractory acute lymphoblastic leukemia: a Therapeutic Advances in Childhood Leukemia Consortium study. J Clin Oncol. 2010 Feb;28(4):648-54.

7 Bertaina A, Vinti L, Strocchio L, Gaspari S, Caruso R, Algeri M, et al. The combination of bortezomib with chemotherapy to treat relapsed/refractory acute lymphoblastic leukaemia of childhood. Br J Haematol. 2017 Feb; 176(4):629-36.

8 Alshiekh-Nasany R, Douer D. L-Carnitine for Treatment of Pegasparaginase-Induced
Hepatotoxicity. Acta Haematol. 2016;135(4): 208-10.

9 Niewerth D, Kaspers GJ, Jansen G, van Meerloo J, Zweegman S, Jenkins G, et al. Proteasome subunit expression analysis and chemosensitivity in relapsed paediatric acute leukaemia patients receiving bortezomib-containing chemotherapy. J Hematol Oncol. 2016 Sep; 9(1):82.

10 Horton TM, Lu X, O'Brien MM, et al. Bortezomib reinduction therapy improves response rates in pediatric ALL in first relapse: A Children's Oncology Group (COG) study (AALL07P1). American Society of Clinical Oncology, Annual Meeting. Chicago, IL, 2013, J Clin Oncol Volume 31 (suppl).

11 Zhao J, Wang C, Song Y, Liu Y, Fang B. Treatment of refractory/relapsed adult acute lymphoblastic leukemia with bortezomib-based chemotherapy. Int J Gen Med. 2015 Jun;8: 211-4. 